\title{
Fantastique médiéval et fantastique potockien
}

\section{Alain Corbellari}

\section{(2) OpenEdition}

\section{Journals}

Édition électronique

URL : http://journals.openedition.org/edl/453

DOI : $10.4000 /$ edl. 453

ISSN : 2296-5084

\section{Éditeur}

Université de Lausanne

\section{Édition imprimée}

Date de publication : 15 décembre 2012

Pagination : 43-58

ISBN : 978-2-940331-29-1

ISSN : 0014-2026

\section{Référence électronique}

Alain Corbellari, «Fantastique médiéval et fantastique potockien », Études de lettres [En ligne], 4 | 2012, mis en ligne le 15 décembre 2015, consulté le 22 décembre 2020. URL : http://

journals.openedition.org/edl/453; DOI : https://doi.org/10.4000/edl.453 


\section{FANTASTIQUE MÉDIÉVAL ET FANTASTIQUE POTOCKIEN}

Aucune trace directe d'une lecture d'une œuvre de la littérature française médiévale n'est décelable chez Potocki. Néanmoins, en comparant le Manuscrit trouvé à Saragosse à des œuvres du Moyen Age (essentiellement ici le fabliau des Trois dames de Paris et le très étrange roman arthurien de Perlesvaus), on apporte aux relations de Potocki avec le fantastique un nouvel éclairage qui rejaillit à son tour sur les œuvres médiévales qui ne sont pas forcément moins imprégnées de fantastique, au sens large, que les romans de l'époque moderne.

Potocki, nous dit-on, avait tout lu. Pourquoi donc, dans ces conditions, n’aurait-il pas été influencé par la littérature médiévale ? ${ }^{1}$ Enoncée ainsi, la question ne peut manquer d'apparaître quelque peu gratuite; cependant, même si les traces certaines d'une imprégnation consciente sont indémontrables, une telle enquête peut nous mener à des considérations bien plus intéressantes qu'un toujours fastidieux relevé des «sources et influences" qui, au fond, n'explique jamais rien des moteurs secrets de la création artistique. Ce qui pourrait en effet se jouer dans le rapport du Manuscrit trouvé à Saragosse avec la littérature française médiévale n'est peut-être autre que la question même du fantastique: trop vite dénié aux ouvrages médiévaux ${ }^{2}$, trop libéralement accordé au chef-d'œuvre de

I. Quant à la vision que Potocki avait de l'histoire et de la civilisation médiévales, nous sommes bien renseignés grâce au récent article de D. Triaire, «Le Moyen Age de Jean Potocki». Mais c'est de littérature que nous aimerions parler ici.

2. Les choses ont heureusement un peu changé - du moins pour les médiévistes - depuis la parution de l'ouvrage fondamental de F. Dubost, Aspects fantastiques de la littérature française médiévale. La thèse de Dubost est qu'il existe bien, quoique généralement très sporadiquement, des moments de «fantastique» dans la littérature 
Potocki ${ }^{3}$, le fantastique est traditionnellement considéré comme ce qui sépare radicalement la production littéraire du Moyen Age de celle de l'aube du romantisme, alors que c'est sans doute précisément en revisitant cette notion que l'on parvient à tisser entre les deux époques un parallèle qui, aussi surprenant qu'il puisse paraitre, éclaire d'un nouveau jour l'histoire longue de la littérature française.

Commençons par rappeler que le XVIII e siècle, on l'oublie trop, a connu, en France, essentiellement dans sa seconde moitié, un grand mouvement d'exhumation de la littérature du Moyen Age: débutant avec le Roman de la Rose, édité en 1732 par Jean-Baptiste Lenglet du Fresnoy, se poursuivant en 1742 avec la publication des chansons de Thibaut de Champagne par Levêque de la Ravellière, il produit son expression la plus célèbre en 1756, avec les trois volumes des Fabliaux et contes de Barbazan, suivis, en 1781, par un volume à même vocation de Le Grand d'Aussy ${ }^{4}$. Parallèlement, La Curne de Sainte-Palaye se faisait le déchiffreur infatigable des manuscrits de la Bibliothèque royale ${ }^{5}$, et le comte de Tressan, animateur, de 1775 à 1789, avec Paulmy et Bastide, de la Bibliothèque universelle des romans, donnait, par ses adaptations (surtout de la littérature arthurienne) le coup d'envoi au «style troubadour " ${ }^{6}$. Enfin, la "Bibliothèque bleue» de Troyes continuait de répandre les adaptations d'une poignée de chansons de geste, parmi lesquelles celle des Quatre fils Aymon, dont le héros, Renaut de Montauban, avait, par ailleurs, connu la fortune que l'on sait grâce au Roland furieux de l'Arioste.

Malgré la diversité des matières renflouées, la teinte volontiers ironique donnée même à des textes qui, a priori, en semblaient dépourvus, faisait

médiévale, c'est-à-dire des moments où l'on ne peut déterminer si la «merveille» est d'origine divine ou diabolique et où cette hésitation est donc génératrice d'angoisse.

3. F. Rosset et D. Triaire ont insisté avec raison sur ce qu'une lecture du Manuscrit trouvé à Saragosse en clé fantastique avait de réducteur, voire de contre-productif (MTS1804, «Présentation», p. 32 sq.).

4. P.-J.-B. Le Grand d'Aussy, Fabliaux ou Contes du XII et du XIII siècle. Sur cet auteur, voir G. Wilson, A Medievalist in the Eighteenth Century.

5. Il allait en tirer la matière du tout premier dictionnaire de l'ancien français, lequel n'allait malheureusement paraître qu'un siècle après sa mort. La Curne avait cependant publié en 1759 des Mémoires sur l'ancienne chevalerie considérée comme un établissement politique et militaire. Sur cet auteur, voir L. Gossman, Medievalism and The Ideologie of the Enlightenment.

6. Voir H. Jacoubet, Le Comte de Tressan et les origines du genre troubadour. 
du fabliau l'hypergenre subsumant le plus communément, en ce siècle réputé frivole qui s'y reconnaissait volontiers, les œuvres inspirées ou récupérées du Moyen Age. A la faveur d'un rapprochement entre la Gaule et le verbe "gauler» ("attraper», avec des connotations volontiers grivoises), la "gauloiserie" avait été reconnue comme l'un des caractères les plus vénérables et les plus certains de la culture française et même les romans courtois (les adaptations de Tressan en témoignent) étaient contaminés par cette désinvolture souriante qui, aux yeux de l'Ancien Régime, caractérisait la littérature du Moyen Age. Il est, de fait, à noter que les partisans de cette vieille littérature, ces thuriféraires des "Antiquités françaises» qui, depuis la fin du XVI $\mathrm{X}^{\mathrm{e}}$ siècle, contestaient le monopole des Antiquités gréco-romaines, se recrutaient essentiellement dans les rangs des intellectuels conservateurs, opposés aux Lumières. De fait, à lire les textes médiévaux modernisés sans vergogne et presque sans y penser au XVIII e siècle, on mesure la proximité que la noblesse ressentait alors avec cette vieille littérature - enfance d'un art sans doute encore en phase avec les idéaux d'une société qui n'avait pas totalement renoncé à ses désirs de panache et d'aventure, mais aussi à sa liberté de mœurs. Le terme "fabliau", à la fin du XVIII e siècle, était devenu à la mode, comme en témoigne le titre d'un recueil de nouvelles de Sade ${ }^{7}$, textes qui n'ont pourtant pas grand-chose à voir avec les vieux "contes à rire» du XIII ${ }^{\mathrm{e}}$ siècle...

Il apparaît donc évident qu'un lettré informé du tournant des Lumières n'a pu qu'être imprégné, fût-ce à son corps défendant, de littérature du Moyen Age. Pourtant, le mot «fabliau» n'apparaît nulle part dans le Manuscrit trouvé à Saragosse, et aucune des multiples intrigues qui s'y enchâssent ne s'appuie sur une trame médiévale reconnaissable, sinon à travers des avatars issus de la Renaissance, de l'âge baroque ou encore des Mille et une nuits, si archétypiques que leur relevé ne nous apporterait rien: faire remonter au Moyen Age les motifs, par exemple, de la jeune fille prisonnière dans la tour, du jeune homme naïf partant à l'aventure ou du trésor trouvé dans une grotte est une banalité qui ne nous explique nullement le sens que prennent ces topoï dans l'œuvre de Potocki.

Il faut donc aborder la question par un autre biais. Nous partirons de l'une des images les plus fortes du roman, celle du réveil du héros sous le gibet de Los Hermanos. Cette scène rappelle évidemment les nombreux

7. Voir B. Didier, «L'image du Moyen Age chez Sade». 
contes merveilleux où un héros se réveille à un endroit tout différent - et généralement moins réjouissant - de celui où il s'était endormi la veille: ainsi, dans Le Bel Inconnu, roman du XIII siècle, le héros, après une nuit d'amour, se réveille-t-il «en un bois ${ }^{8}$; à la même époque le prédicateur Etienne de Bourbon nous raconte l'histoire d'un paysan qui rencontre une jeune fille accorte, la suit dans son lit et se réveille le demain "gisant pitoyablement sur son fagot et berné" ${ }^{9}$ : le motif semble donc topique. C'est cependant à sa réécriture ironique ${ }^{10}$ que j’aimerais m'attacher ici, la scène du Manuscrit ayant en effet toujours irrésistiblement évoqué en moi le souvenir d'un fabliau du XIV $\mathrm{X}^{\mathrm{e}}$ siècle, Les Trois dames de Paris, dont l'intrigue n'est presque en rien comparable à celle de l'épisode potockien, mais qui le rejoint cependant par la bizarrerie macabre de son parcours et l'impression d'«inquiétante étrangeté» qui en émane.

Ce fabliau de Watriquet de Couvin est précisément datable, ce qui est exceptionnel pour un texte de ce type; il est en effet dit explicitement se dérouler en 1320 , ce qui le situe dans la phase d'épanouissement terminal de ce genre disparu au milieu du XIV siècle sans laisser la moindre descendance directe (sinon à l'étranger dans les contes de Boccace et de Chaucer). Mais ce point d'histoire littéraire est moins important que l'intention révélée par cette mention de date qui, selon toute évidence, vise à rapprocher l'intrigue du présent du lecteurauditeur; tout se passe ici comme si le narrateur des Trois dames de Paris voulait absolument nous faire croire qu'il nous raconte un fait divers authentique. Autrement dit, un tel récit, dont le sel réside en grande partie dans l'affirmation qu'il est réellement arrivé, appartient, dans la communication orale, au registre de la rumeur, ou, pour l'exprimer par un terme aujourd'hui à la mode, de la «légende urbaine» (registre auquel semblait il est vrai déjà appartenir l'exemplum pseudo-féerique d'Etienne de Bourbon). Corollairement, sur un plan plus strictement littéraire, on observera que le pacte de lecture, basé sur la véridiction, que nous propose l'auteur est déjà celui des romanciers modernes. Alors que le fabliau, conformément à son étymologie (= "petite fable»), commence volontiers par brouiller les pistes référentielles, en nous assurant que l'histoire qu'il

8. Le Bel Inconnu, roman d'aventure, v. 5399.

9. Cité par L. Guyénot, La mort féerique, p. 158.

Io. S'il est vrai, comme l'a bien montré F. Rosset dans "Le langage du fantastique", que le fantastique se construit toujours déjà à travers l'ironie et la réécriture. 
nous conte est arrivée «il y a bien longtemps», Watriquet, au contraire, n'hésite pas à sous-entendre que l'on trouvera sans difficulté des témoins pour le confirmer. Mais, comme dans la légende urbaine, on ne pourra jamais remonter qu'à l'ami d'un ami d'un ami qui... bref à un "on" aussi impersonnel qu'introuvable!

Les Trois dames de Paris nous raconte donc l'histoire de trois joyeuses commères qui, ayant décidé de faire une virée tardive dans une taverne où l'on danse, boivent et se démènent tant qu'elles en tombent ivres mortes et complètement nues au milieu de la rue. Enterrées par des édiles qui les ont crues décédées, elles se réveillent le lendemain dans le cimetière des Innocents (!). Se relevant à grand-peine, elles parcourent ainsi, couvertes de boue et d'immondices, les rues de la capitale telles de véritables mort(e)s-vivant(e)s.

On voit tout de suite l'analogie que l'on peut faire entre le fabliau et le texte de Potocki: dans les deux cas, il y a brutale succession d'une nuit d'ivresse (amoureuse ou bachique) et d'un dur réveil parmi les morts. Poser le problème en termes d'influences ne nous mènerait nulle part: trop d'éléments distinguent fondamentalement les deux textes. Pourtant, leur mouvement est pareil : le plaisir attendu débouche, après un moment d'inconscience, sur une vision grimaçante et douloureuse, métaphores, si l'on y tient, de la xylostomie dans le fabliau et de la tristesse post-coïtale dans le Manuscrit trouvé à Saragosse.

Une différence importante distingue cependant ces deux scènes: ce qui frappe chez Potocki, mais qui est absent du fabliau, c'est évidemment l'ellipse, favorisée, il est vrai, chez l'écrivain polonais, par la structuration du texte en "journées». Rien n'annonce, à l'issue de la première nuit, de quoi sera fait le lendemain, et Potocki se donne même les gants de retarder de quelques lignes la macabre découverte de son héros, les premières phrases de la «seconde journée» mimant précisément le processus d'un réveil qui ne révèle que progressivement l'inconfort de la situation dans laquelle se trouve Alphonse van Worden:

Enfin je me réveillai réellement; le soleil brûlait mes paupières, je les ouvris avec peine, je vis le ciel, je vis que j'étais en plein air, mais le sommeil appesantissait encore mes yeux. Je ne dormais plus, mais je n'étais pas encore éveillé. Des images de supplices se succédèrent les unes aux autres, j'en fus épouvanté. Je me soulevai en sursaut et me mis sur mon séant... 
Où trouverai-je des termes pour exprimer l'horreur dont je fus alors saisi?... J'étais couché sous le gibet de Los Hermanos ${ }^{11}$.

La première impression désagréable ("le soleil brûlait mes paupières») pourrait encore ne signaler qu'un rayon traversant une fenêtre et n'avoir rien de plus traumatisant que l'ennui de voir l'inquiétude du jour succéder au calme de la nuit. La conscience du plein air est déjà plus inquiétante, mais les impressions franchement hostiles n'appartiennent d'abord qu'au domaine du cauchemar. La rupture de paragraphe, enfin, désigne l'irruption véritable de "l'horreur" dans la réalité, et la reconnaissance du lieu sonne, comme un glas, l'impossibilité de l'échappatoire dans l'illusion. Un tel texte, à proprement parler, n'entretient que des liens minimaux avec l'idée du fantastique. En effet, si celui-ci réside bien, comme le veut Todorov, dans "l'hésitation", cette dernière n'aura duré que le temps d'un réveil légèrement laborieux, et rien dans la suite du récit ne viendra remettre en doute la certitude acquise: notre héros s'est bien réveillé sous le gibet de Los Hermanos! Libre au lecteur, à partir de là, d'imaginer comment Alphonse a pu se retrouver à cet endroit après une tendre nuit d'amour; mais, à coup sûr, les explications rationnelles ne font pas défaut pour expliquer sa translation: une nuit suffit largement à mettre en scène cette farce de mauvais goût faite à un jeune homme trop sûr de lui dont on aura abusé du sommeil et de la crédulité (sans compter que les jeunes filles de la veille pouvaient déjà n'avoir existé qu'en songe...). On n'exclura certes pas la possibilité que les séductrices aient été en réalité des stryges et que le déplacement se soit fait par téléportation, mais rien, dans le texte de Potocki, ne nous oblige à privilégier cette dernière solution ${ }^{12}$.

Le fabliau des Trois dames de Paris semble de ce point de vue beaucoup plus simple puisqu'il est en grande partie le récit d'une dégradation annoncée. On nous raconte en effet par le menu comment les femmes boivent jusqu'à plus soif, dansent, se déshabillent pour être plus à l'aise, confient leurs habits au tavernier (qui prendra bien soin de ne pas les

II. $M T S-1804$, p. 86.

I2. Comme le dit très bien François Rosset dans «Un roman fantastique?», p. 267 : "les histoires du Manuscrit trouvé à Saragosse ne laissent jamais longtemps le lecteur dans l'incertitude relativement aux propriétés de monde représenté; c'est principalement pour cette raison que le fantastique s'y trouve bien vite neutralisé». 
leur rendre), sortent nues, se vautrent dans la boue et s'écroulent finalement au beau milieu de la rue. Ainsi, leur découverte par les passants ne signale pas la fin mais bien le début du mystère:

La jurent a mout grant vilté,

l'une sus l'autre comme mortes,

tant que par tout guichez et portes

de la cité furent ouvertes,

c'on vit les merveilles apertes.

Elles gisaient là dans le plus misérable état, l'une sur l'autre, comme si elles étaient mortes, si bien qu'au moment où les guichets et les portes de la cité furent ouverts, les merveilles apparurent dans toute leur évidence ${ }^{13}$.

Pour ne pas charger d'entrée de jeu le texte d'une connotation intempestive, il conviendrait peut-être de rendre au mot merveilles son sens littéral de «spectacle étonnant». Cependant, l'expression «merveilles apertes" est sans nul doute un clin d'œil à la littérature arthurienne, si bien que son acception courante se trouve, aux yeux d'un lecteur du $\mathrm{XIV}^{\mathrm{e}}$ siècle, déjà revêtue d'une aura la tirant nettement vers ce que nous appelons aujourd'hui le "merveilleux" stricto sensu. Et la suite du texte nous confirme qu'il n'y a en effet pas seulement là une curiosité pour les badauds: si le narrateur joue abondamment de l'aspect prosaïque du spectacle, n'hésitant pas à dire que les femmes gisent "comme merdes en mi la voie» (v. 209), que les maris accourus sont courroucés de voir découvertes les "cus et testes» de leur épouse (v. 219) et que le vin leur sortait "par les gencives [...] et par tous les conduis» (v. 226-227), il dispose en même temps les éléments qui permettront de faire apparaître une «merveille» plus grande encore: la «résurrection» des trois soûlardes.

Celles-ci se réveillent à minuit, inversant donc l'ordre normal du passage de la vie à la mort. Certes, "elles n'odorent pas encens» (v. 234), mais, surtout, elles provoquent la terreur sur leur passage, et le fossoyeur résume l'opinion générale en les prenant pour des revenantes tout droit sorties de l'Enfer:

Et quant ilec les a trouvees, de grans merveilles s'en seigna,

I3. Watriquet de Couvins, Les Trois dames de Paris, p. 124 (v. 184-188). 
et dist: «Dyables les engigna

qui les a raportees ci.

Oiés, seigneur, pour Dieu merci, comment sont elles revenues?» ${ }^{14}$

Les rencontrant à cet endroit, il se signa devant ces grandes merveilles et dit: "C'est le Diable qui les a enchantées et rapportées ici. Ecoutez donc, messieurs-dames, pour l'amour de Dieu, comment ont-elles fait pour revenir?»

La reprise du terme "merveilles", l'allusion aux ruses du Diable ("engigner" signifie littéralement "tromper») et au statut de "revenantes" de ces apparitions nous installent au cœur de la superstition médiévale; mais l'auteur n'est pas dupe, signe que la croyance aux fantômes ne saurait en aucun cas être constitutive d'une introuvable "mentalité» d'époque. Elle est même si peu partagée que les auteurs de fabliaux sont unanimes à contester la réalité des manifestations de l'irrationnel dans le quotidien: les fabliaux des Trois bossus ménestrels et d'Estormi se moquent ainsi de personnages qui croient leur femme sans barguigner lorsque, pour se débarrasser respectivement de trois bossus et de trois prêtres morts accidentellement mais dans une position compromettante pour elles, les commères ont jugé plus prudent de faire croire à leur mari qu'il n'y avait qu'un seul cadavre, mais qu'il était revenu deux fois. La ruse marche si bien que, sitôt le troisième larron enterré, le mari, resp. le portefaix, estourbit un quatrième personnage qui n'avait pour seuls torts que de passer par là par hasard et d'afficher les mêmes caractères (un bossu / un prêtre) que les trois précédents. On pourrait relever, dans les fabliaux, bien d'autres exemples de dérision de la croyance aux fantômes ${ }^{15}$; le texte qui, de ce point de vue, va le plus loin est celui du Sacristain, dont les nombreuses versions multiplient les avanies subies par le cadavre d'un prêtre tour à tour caché, dérobé, échangé, installé sur un cheval tel un cavalier de l'Apocalypse, et provoquant à chaque fois la terreur de naïfs qui croient que ses disparitions ou déplacements incongrus témoignent de son statut de revenant ${ }^{16}$. Et si, par exception,

I4. Watriquet de Couvins, Les Trois dames de Paris, p. 126 (v. 270-275).

I5. On peut citer les fabliaux de Barat et Haimet, des Tresses ou encore du Chevalier à la robe vermeille.

I6. Pour un aperçu complet des fabliaux, voir le Nouveau recueil complet des fabliaux. 
le merveilleux est utilisé dans un fabliau, c'est pour l'unique raison qu'il permet des grivoiseries inédites: ainsi du Chevalier qui fit parler les cons, dont le titre se passe de commentaire (c'est le prototype de l'histoire des Bijoux indiscrets) ou du Mantel mautaillé qui révèle, par son inadaptation aux formes du corps, l'infidélité des femmes.

Dans tous ces textes, si fantastique il y a, il ne peut donc être considéré que du seul point de vue des personnages: auteurs ni narrateurs ne sont jamais dupes, mais ils se plaisent à mettre en scène l'«hésitation» et, plus souvent encore, la terreur d'acteurs internes à la diégèse. De ce point de vue, le parallèle avec Potocki s'avère pertinent car, si l'on ne peut nier la réception "fantastique» du Manuscrit trouvé à Saragosse, illustrée en particulier par Roger Caillois, la critique actuelle est, comme on l'a rappelé, beaucoup plus circonspecte face à cette lecture trop partielle; et il se pourrait bien que Potocki, à l'instar des auteurs des fabliaux, installe le sentiment d' «inquiétante étrangeté » à l'intérieur de ses personnages bien davantage qu'il ne tente de l'inspirer à ses lecteurs. Lors de la dixième journée, par exemple, Alphonse refuse de prendre pour argent comptant les étranges déclarations de Pascheco, se disant que ce dernier "pouvait être devenu borgne par un tout autre accident que par sa liaison amoureuse avec les deux pendus, et [que] son effroyable histoire pouvait être un conte " ${ }^{17}$. Plus caractéristique encore, et nous avons là affaire à une intrigue véritablement digne d'un fabliau, même si aucun texte médiéval n'en reproduit exactement l'anecdote: l'épisode des prétendus spectres dans le cimetière ${ }^{18}$ connaît une conclusion (ce n'étaient que des étudiants en médecine volant des cadavres pour leur maître), qui met en valeur des qualités qui sont par excellence celles des héros des contes à rire médiévaux: la ruse et le sens de la mystification. Le matérialisme du fabliau médiéval se retrouve ainsi, et de manière peut-être inattendue, totalement en phase avec le scepticisme ironique des Lumières finissantes.

Cependant, le fantastique potockien ne saurait tout à fait se réduire à un pur jeu avec les angoisses de ses personnages: squelettes, fantômes, gnomes, et autres personnages extraordinaires tel le Juif errant, ne sont

17. MTS-1804, p. 198.

I8. MTS-1804, vingt-sixième journée, p. 440. On pourrait à l'extrême rigueur songer au roman arthurien anonyme du XIII' siècle L'Atre périlleux, qui commence par le spectacle de diables se battant dans un cimetière (d'où le titre: aîtres = "cimetière"). 
pas toujours aussi facilement "dégonflés». Un épisode, en particulier, doit être mentionné ici: celui, à l'intérieur du récit du commandeur de Toralva, où l'on voit des personnages peints descendre de leur cadre et s'entretenir avec le narrateur intra-diégétique ${ }^{19}$. Or ces portraits d'où sortent des fantômes sont précisément dits remonter... au Moyen Age! ${ }^{20}$ Ici encore, cependant, le réveil du narrateur - quoique moins clairement que dans la scène du gibet - est de nature à nous rassurer: alors qu'il était certain d'avoir été blessé par l'un des fantômes, le commandeur se réveille le lendemain «sans aucune blessure» (p. 553).

S'il n'est pas vraiment surprenant de voir un écrivain du début du XIX siècle ancrer une ambiance d'inspiration "gothique» dans des souvenirs prétendument médiévaux, le fait mérite tout de même d'être souligné, s'agissant d'un roman dont les sources sont si diverses, car il nous rappelle opportunément la profonde ambivalence dont les récits du Moyen Age pouvaient être l'objet au tournant des Lumières, apparaissant à la fois comme source de comique (l'esprit des fabliaux) et d'inquiétude. De fait, ce que Francis Dubost a, voici deux décennies, identifié comme le fantastique médiéval dépasse largement la dérision des contes à rire pour plonger bien plus profondément dans les ambiguïtés du merveilleux breton ${ }^{21}$. Et à cet égard, la comparaison des scènes du gibet de Los Hermanos et des portraits animés avec l'épisode initial de l'étrange et inquiétant (et ce n'est pas une formule de style!) roman médiéval de

19. MTS-1810, trente-septième journée, p. 549-554.

20. Petite remarque en passant à l'intention des éditeurs du Manuscrit: il est inexact de prétendre que le mot manzier est absent des dictionnaires d'ancien français (MTS1810, p. 550), du moins si l'on estime - ce qui est plus que vraisemblable - que l'on a affaire là à une forme de manzere que La Curne de Sainte-Palaye (Dictionnaire historique de l'ancien langage françois, t. VII, p. 269, col. 2), cite à partir d'une glose d'un texte latin de 1603 sur les personnages «nez d'une femme publique et d'un père incertain». La forme légèrement différente que lui donne Potocki semble attester que le mot était connu dans les milieux érudits de son temps. Mais de fait, ce n'est pas un mot d'ancien français! Le $F E W$, sous *mandia- (t. VI, 1, 1969, p. 155, col. 2) répertorie bien un manzere, régionalisme pour désigner une vache stérile, mais le sens attesté par La Curne et Potocki reste introuvable dans les dictionnaires modernes de l'ancienne langue.

2I. Le fait que sur les quelque mille pages de son ouvrage on ne trouve que des allusions presque insignifiantes aux fabliaux montre bien à quel point ce corpus est périphérique dans la problématique du fantastique médiéval. 
Perlesvaus pourrait donner une tout autre dimension à la confrontation que nous proposons ici.

Probablement écrit dans la première moitié du XIII ${ }^{\mathrm{e}}$ siècle ${ }^{22}$, Perlesvaus ou Le Haut Livre du Graal est une réécriture en prose, très libre, du mythe du Graal, qui privilégie les aspects noirs, ténébreux et maléfiques de l'univers arthurien ${ }^{23}$. Sa scène la plus étonnante est peutêtre celle sur laquelle il s'ouvre; le chevalier Cahus, au sortir d'un rêve angoissant, se réveille en appelant à l'aide:

Li vallez fu cochiez ainsi com ge vos di. E o premerain some qu'il fu endormiz, li sanbloit que li rois en ert alez sanz lui. [Suit un long récit de rêve, très détaillé et très éloigné des poncifs médiévaux du genre. Cahus s'y bat avec "un home noir et let»:] E li valez fiert des eperons, e cuide celui otrer; et cil le haste, e le fiert du cotel o destre costé si qu'il ienbat o cors desq'enz o manche. Li vallez, qui gisoit en la sale a Carduell, qi ce ot songié, s'esveilla e cria a haute voiz: «Sainte Marie! le provoire! Aidiez, aidiez, car ge sui morz!»

L'écuyer s'était couché comme je vous le dis et une fois endormi, dans son premier sommeil, il rêva que le roi s'en était allé sans lui. [...] l'écuyer piqua des éperons, croyant pouvoir passer outre, mais son adversaire le serra de près et le frappa avec son couteau au flanc droit, si fort qu'il le lui enfonça dans le corps jusqu'au manche. L'écuyer, qui était couché dans la grande salle à Cardueil, qui avait rêvé tout cela, se réveilla et cria à haute voix: «Sainte Marie! Un prêtre! Au secours, au secours, je suis mort!» 24

La scène est forte, mais le lecteur ne voit de prime abord guère de raison d'être inquiet pour Cahus: combien de fois nous sommes-nous réveillés

22. Le débat sur sa datation n'est pas clos: s'agit-il d'un texte précoce (v. 1210) dont les archaïsmes témoignent d'une réécriture du mythe non influencée par sa canonisation dans le grand cycle du Lancelot Graal (v. 1220-1230) ou d'une réélaboration tardive (après 1240) prenant délibérément le contre-pied d'une vulgate bien établie? Au vu de la virtuosité dont témoigne l'auteur du Perlesvaus, l'auteur de ces lignes avoue être tenté par la deuxième solution.

23. Comme le dit F. Dubost (Aspects fantastiques de la littérature française médiévale, p. 779): «le texte laisse donc percevoir un certain flottement dans la distribution du bien et du mal, une indécision sur l'appartenance de certains êtres, une part imprévisible dans les comportements".

24. Le Haut Livre du Graal, p. 136-139. 
encore captifs des illusions d'un cauchemar? Mais Cahus n'est pas victime d'une illusion, ou d'une "fascination» comme dit le commandeur de Toralva: il a réellement dans le flanc - et qui plus est au flanc gauche, alors qu'il s'agissait dans le rêve du flanc droit... - le couteau dont on l'a blessé en rêve! Et comme il va mourir de cette blessure, il n'y a nulle atténuation possible à notre étonnement; nous nous trouvons bien face à l'un des très rares épisodes de la littérature narrative médiévale où la notion de fantastique peut être appliquée dans le sens exact qu'on lui donne lorsque l'on parle des textes de l'époque romantique ${ }^{25}$.

Ici l'influence sur Potocki, déjà peu probable dans le cas des fabliaux, est à exclure totalement, le Perlesvaus n'ayant été exhumé qu'au $\mathrm{XIX}^{\mathrm{e}}$ siècle; et encore une fois la comparaison directe n'apporterait rien. Tout au plus peut-on constater une similitude de construction entre les épisodes de la mort de Cahus et des réveils pénibles des héros du Manuscrit trouvé à Saragosse: tout tourne toujours autour d'une rupture narrative inexplicable provoquant une dysphorie - rapidement surmontable chez Potocki, fatale dans le Perlesvaus - annoncée par une angoisse onirique. Mais cette fois-ci, contrairement à ce que nous observions dans Les Trois dames de Paris, le texte médiéval dépasse dans le fantastique le Manuscrit trouvé à Saragosse: si l'explication rationnelle restait possible (et même plutôt satisfaisante) dans le texte potockien, elle ne l'est plus dans le Haut Livre du Graal: la mort de Cahus transgresse les frontières du rêve et de la réalité et ni Dieu ni diable ne semblent suffire à expliquer cette disjonction qui, à l'intérieur de la fiction, c'est-à-dire au-delà du monde où l'existence du surnaturel peut (voire doit) être acceptée, joue avec les catégories mêmes de l'effectif et du virtuel ${ }^{26}$. Ce fantastique-là,

25. Ce n'est pas pour rien que F. Dubost réserve pour le dernier chapitre de sa vaste étude (Aspects fantastiques de la littérature française médiévale, p. 777-807) l'analyse du Perlesvaus, dont il rappelle, sans infirmer ce jugement, qu' "on a souvent parlé de fantastique» à son propos (p. 777). Voir aussi, sur le rêve de Cahus: M. Zink, «Le Rêve avéré».

26. En fait, il y aurait bien un moyen de rationaliser l'histoire de Cahus, mais il n'est sans doute guère à la portée d'une imagination médiévale ( $\mathrm{M}$. Zink, par contre, en joue quelque peu dans son roman arthurien Déodat ou la transparence, qui raconte précisément les aventures d'un frère de Cahus): ce serait de considérer cette scène comme le début d'un roman policier, où l'on finirait par découvrir que c'est bien et uniquement dans la réalité que Cahus a été poignardé, le rêve ayant été directement provoqué par le coup et n'ayant instantanément déroulé qu'une durée fictive, selon le fameux principe du «rêve de la guillotine» glosé par Freud dans la Traumdeutung. 
que l'on retrouvera chez Washington Irving, Théophile Gautier ou encore H. P. Lovecraft, n'est pas celui de Potocki.

Nous aboutissons donc à la conclusion que le fantastique médiéval, même s'il n'est souvent le fait que de moments narratifs assez ponctuels, n'a rien à envier à celui d'un auteur du début du XIX ${ }^{\mathrm{e}}$ siècle. Potocki, en fin de compte, partage bien davantage la vision démystificatrice du surnaturel que promeuvent les auteurs de fabliaux que les angoisses terrifiantes du narrateur du Perlesvaus. Mais le fait qu'il se situe au seuil d'une période où le fantastique va connaître une vogue sans précédent nous le fait évidemment voir avec d'autres yeux; a-t-il, pour autant, plus de mérite à être encore adepte des Lumières, c'est-à-dire à résister à la montée de l'irrationnel romantique que n'en ont les auteurs de fabliaux à oser braver les croyances de leur temps, autrement dit, en clair, à contrecarrer les menées de ceux qui tirent profit de la crédulité ambiante? ${ }^{27}$ Que Potocki soit plus jeune de cinq ou six siècles que ses devanciers médiévaux ne signifie donc pas qu'il serait plus avancé qu'eux sur le chemin de la dérision ou sur celui de la terreur. Simplement, et au-delà des déterminations de l'histoire et de la sociologie littéraires, il suit sa propre voie, pour notre plus grand plaisir, et pour le sien, nous prouvant par son exemple que chaque auteur jouit toujours d'une certaine marge de liberté pour réélaborer à son seul usage des catégories de pensée et des interrogations sur la réalité et la logique de notre monde qui - au-delà des déterminations pesant à toutes les époques de la littérature sur les esprits sans imagination - peuvent être repensées à nouveaux frais ${ }^{28}$.

Alain Corbellari

Universités de Lausanne et de Neuchâtel

27. Comme le dit fort bien Dubost (Aspects fantastiques de la littérature française médiévale, p. 199): «le narrataire construit par le texte des fabliaux en question est invité à participer à un scénario à travers lequel se liquide, sous une forme généralement ludique, le conflit permanent entre la jouissance et la loi».

28. Cette idée d'une histoire longue du fantastique rejoint les considérations de F. Rosset dans «Le langage du fantastique». 


\section{BIBLIOGRAPHIE}

\section{Textes}

Le Bel inconnu, roman d'aventure, éd. par G. Perrie Williams, Paris, Champion, 1983.

Le Haut Livre du Graal. (Perlesvaus), éd. et trad. par A. Strubel, Paris, Le Livre de poche, 2007 (Lettres gothiques).

Le Grand d'Aussy, Pierre-Jean-Baptiste, Fabliaux ou Contes du XII et du XIII e siècle. Fables et romans du XIII e siècle, Paris, Onfroy, 1781 ; rééd.: Genève, Slatkine reprints, 1971.

MTS-1804 et MTS-1810 = Ротоскі, Jean, Manuscrit trouvé à Saragosse (version de 1804 et version de 1810), éd. par François Rosset, Dominique Triaire, Paris, Flammarion, 2008 (GF 1342-1343).

Nouveau recueil complet des fabliaux, 10 vols, éd. par W. Noomen, N. Van den Boogard, Assen, Van Gorcum, 1983-1998.

Watriquet de Couvins, Les Trois dames de Paris, in Fabliaux français du Moyen Age, t. I, éd. par Philippe Ménard, Genève, Droz, 1979 (Textes littéraires français).

Zink, Michel, Déodat ou la transparence: un roman du graal, Paris, Seuil, 2002.

\section{Travaux}

Didier, Béatrice, "L'image du Moyen Age chez Sade», La Licorne, 6 (1982), t. II, p. 301-312.

Duвоsт, Francis, Aspects fantastiques de la littérature française médiévale, 2 vols, Paris, Champion, 1991.

Gossman, Lionel, Medievalism and The Ideologie of the Enlightenment. The World and Work of la Curne de Sainte Palaye, Baltimore, Johns Hopkins Press, 1968. 
Guyénot, Laurent, La mort féerique. Anthropologie du merveilleux XII ${ }_{-}$ $X V^{e}$ siècle, Paris, Gallimard, 2011 (Bibliothèque des histoires).

Jacoubet, Henri, Le Comte de Tressan et les origines du genre troubadour, Paris, PUF, 1923.

La Curne de Sainte-Palaye, Jean-Baptiste, Dictionnaire historique de l'ancien langage françois, Paris, Champion, 1880.

Rosset, François, «Un roman fantastique?», in De Varsovie à Saragosse: Jean Potocki et son auvre, éd. par François Rosset, Dominique Triaire, Louvain, Peeters, 2000, p. 253-268.

—, "Le langage du fantastique», Poétique, 166 (avril 2011), p. 203-214.

Triaire, Dominique, "Le Moyen Age de Jean Potocki», Revue des langues romanes, $115 / 1$ (2011), p. 27-44.

Wilson, Geoffrey, A Medievalist in the Eighteenth Century: Le Grand d'Aussy and the "Fabliaux ou Contes", La Haye, Nijhoff, 1975.

Zink, Michel, "Le Rêve avéré. La Mort de Cahus et la langueur d'Arthur du Perlesvaus à Fouke le Fitz Warin", Mélanges René Fromilhague, Littératures, 9-10 (1984), p. 31-38. 
